\title{
Klimaschwankungen und Großwetterlagen in Mitteleuropa seit 1881
}

Die noch zu Beginn unseres Jahrhunderts hervorgehobene Behauptung, klimatische Verhältnisse seien konstant, wurde durch Auswertungen der Meßergebnisse meteorologischer Elemente widerlegt. Statistische Untersuchungen von langjährigen Meßreihen, sogenannten Säkularreihen, konnten Veränderungen des Klimas aufzeigen. Wir sprechen von säkularen Klimaschwankungen und -pendelungen oder auch von instrumentellen Schwankungen. Zahlreiche Klimaindizien bzw. -zeugen biologischer, geomorphologischer, pedologischer und geologischer Art, die Rückschlüsse auf das Klima historischer und geologischer Epochen erlauben, die klassischen Methoden der Warven- und Dendrochronologie zur absoluten Altersbestimmung und paläoklimatische Datierungsmethoden lassen erkennen, daß Klimaschwankungen und Klimaänderungen beträchtlichen Ausmaßes während der gesamten Erdgeschichte vorgekommen sind.

\section{Analyse der Temperaturverhältnisse in Mitteleuropa}

Zum Thema «Klimaschwankungen» liegen bereits eine Reihe von Publikationen vor (u. a. von wU. STELT, J., 1962; RUDLOFF, H.v., 1967; EISENHARDT, T., 1968; LALLA, H., 1976; MEYER, A.-M., 1978). Die hiermit vorgelegte Untersuchung soll deren Ergebnisse ergänzen und einen Beitrag zur historischen Klimakunde von Mitteleuropa leisten. Sie orientiert sich an den von der WMO (1966) vorgelegten Kriterien für Klimaschwankungen und beabsichtigt die Temperatur in Verbindung zu Schwankungen derWetterlagen zu bringen. Erstes Ziel ist die Ermittlung der säkularen Schwankungen derTemperatur der Jahre, Jahreszeiten und Monate in ihrer räumlich-zeitlichen Differenzierung in den letzten 100 bis 200 Jahren.

Probleme bereitete die Auswahl der Beobachtungsstationen - hier spielt die Homogenität der Beobachtungsreihen eine große Rolle - und die Beschaffung und Zusammenstellung des Datenmaterials.

Für die Erfassung der Klimaschwankungen ist die «Warschauer Resolution» vom September 1935 maßgebend. Die Warschauer Resolution bezeichnet ' Klima «die mittleren meteorologischen VerhältMonat und Jahr nach einer Ausgleichung

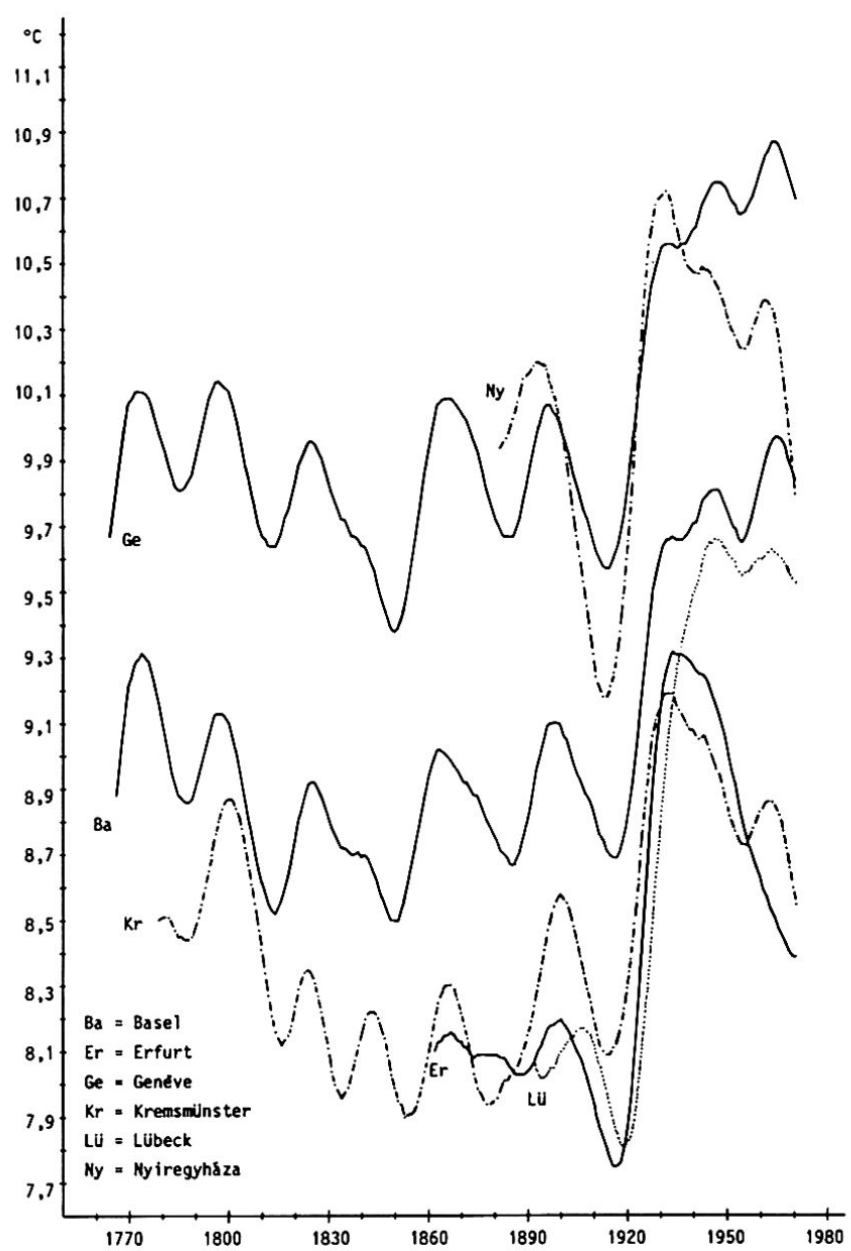

Abb. 1 Lufttemperatur (Herbst)

über dreißig Jahre». Die Differenz zweier 30jähriger Mittel ungeachtet ihrer Größe wird als Klimaschwankung definiert. Mit Hilfe der Gaußschen Tiefpaßfilterung wurden für 42 Stationen die mittleren Lufttemperaturen für Monate, Jahre und Jahreszeiten berechnet und graphisch dargestellt, wie es beispielhaft auf Abbildung 1 zu sehen ist. Die Abbildung zeigt für einige Stationen den säkularen Temperaturverlauf im Herbst.

Seit 1794/1800 setzte im Untersuchungsgebiet eine allgemeine Abnahme der Jahresmitteltemperaturen

Jürgen Grabau, Dr., Kilianstr. 153, D-4790 Paderborn 
Tab. 1 Großwettertypen und Großwetterlagen Europas (nach HESS, P. \& BREZOWSKY, H., 1977, S. 4)

\begin{tabular}{|c|c|c|}
\hline Zirkulationsform & $\begin{array}{l}\text { Großwettertyp } \\
\text { GT }\end{array}$ & $\begin{array}{l}\text { Großwetterlage } \\
\text { GWL }\end{array}$ \\
\hline zonal & West & $\begin{array}{l}\text { Westlage, antizyklonal/zyklonal WaWz } \\
\text { südliche Westlage Ws } \\
\text { winkelförmige Westlage Ww }\end{array}$ \\
\hline gemischt & $\begin{array}{l}\text { Südwest } \\
\text { Nordwest } \\
\text { Hoch Mittel- } \\
\text { europa }\end{array}$ & $\begin{array}{l}\text { Südwestlage, antizyklonal/zyklonal SWa/SWz } \\
\text { Nordwestlage, antizyklonal/zyklonal NWa/NWz } \\
\text { Hoch über Mitteleuropa, Hochdruckbrücke } \\
\text { über Mitteleuropa HM, BM }\end{array}$ \\
\hline meridional & $\begin{array}{l}\text { Nordost } \\
\text { Ost } \\
\text { Südost } \\
\text { Süd }\end{array}$ & $\begin{array}{l}\text { Nordlage, antizyklonal/zyklonal Na/Nz } \\
\text { Hoch-Nordmeer-Island, antizyklonal/zyklonal HNa/HNz } \\
\text { Hoch Britische Inseln HB } \\
\text { Trog Mitteleuropa TrM } \\
\text { Nordostlage, antizyklonal/zyklonal NEa/NEz } \\
\text { Hoch Fennoskandien, antizyklonal/zyklonal HFa/HFz } \\
\text { Hoch-Nordmeer-Fennoskandien, antizyklonal/zyklonal HNFa/HNFz } \\
\text { Südostlage, antizyklonal/zyklonal SEa/SEz } \\
\text { Südlage, antizyklonal/zyklonal Sa/Sz } \\
\text { Tief Britische Inseln TB } \\
\text { Trog Westeuropa TrW }\end{array}$ \\
\hline
\end{tabular}

ein, die sich auf alle Jahreszeiten erstreckte. Bereits um 1850/53 begann die Erwärmung der Frühjahre, danach auch bei den Wintern (1890/91), Herbsten (1914/18) und Sommern (1914/24). Während dieWintertemperaturen bereits nach 1916/17 ihren Höhepunkt überschritten, hielt die Temperaturzunahme der übrigen Jahreszeiten bis 1930/60 an. Zwar kühl. ten sich zunächst die Winter wieder ab und dann die anderen Jahreszeiten, jedoch besteht im Herbst und im Winter immer noch ein Wärmeüberschuß. Im niederländischen, deutsch-schweizerischen und tschechischen Raum sind auch die Sommer noch zu warm.

\section{Gibt es Zusammenhänge zwischen Schwankungen der Temperatur und solchen der allgemeinen Zirkulation?}

Die im Untersuchungsgebiet festgestellten Klimaschwankungen im Verlaufe der letzten 200 Jahre werfen die Frage auf, welche Ursachen diesen Veränderungen im Klimaablauf zugrunde liegen. Bei einer Analyse von Ursache und Wirkung können zur Erklärung der Klimaänderungen etwa der letzten 1000 Jahre eine Reihe von astro- und geophysikalischen Hypothesen wegfallen, da sie nur in geologischen Zeitskalen von $10^{4}$ bis $10^{9}$ Jahren eine Rolle spielen. Dagegen stehen Veränderungen der allgemeinen Zirkulation in Atmosphäre und Ozean in einem engen Zusammenhang mit den in den letzten Jahrzehnten oder Jahrhunderten festgestellten Schwankungen einzelner Klimaelemente. Einige Autoren sehen die Ursache der Klimaschwankungen der letzten Jahre vor allem in der Unstetigkeit der atmosphärischen Zirkulation.
Erste Untersuchungen zu dem Problemkreis «Schwankungen der atmosphärischen Zirkulation» legten A. WAGNER (1929) und R. SCHERHAG (1936) vor. Sie untersuchten anhand zahlreicher Stationen die Änderungen des mittleren Luftdrucks. A. WAGNER fand eine Verstärkung der atmosphärischen Zirkulation im atlantisch-europäischen Raum im Zeitraum 1911/20. R. SCHERHAG konnte eine Zunahme der Westdrift auf der ganzen Nordhalbkugel nachweisen, die sich bis 1921/30 fortsetzte und zu einer Erwärmung des größten Teils der Nordhemisphäre führte. Die Schwankungen der atmosphärischen Zirkulation spiegeln sich auch in langjährigen Änderungen der Häufigkeiten des Auftretens von Großwetterlagen (Tabelle 1) wider. P. HUPFER (1962) und P. HESS \& H. BREZOWSKY (1977) vergleichen die Perioden 1901/30 und 1931/60 hinsichtlich der Häufigkeit des Auftretens europäischer Großwetterlagen. Ein erster Ansatz zur Untersuchung des säkularen Ganges ausgewählter Zirkulationstypen liegt von H. BREZOWSKY (1952) vor.

Hier knüpfen dann Untersuchungen des Verfassers an:

Die Schwankungen der Hauptzirkulationsformen zonal, meridional und gemischt und einzelner Großwetterlagen bzw. Großwettertypen wurden für Jahre und Jahreszeiten untersucht und beschrieben. Als Ausgangsmaterial stand hierfür der Katalog der Großwetterlagen zur Verfügung. Die zunächst ermittelten absoluten Häufigkeiten sind in relative Häufigkeiten umgerechnet und anschließend mit Hilfe der Gaußschen Tiefpaßfilterung bearbeitet worden (vgl. Abb. 2 und 3).

Im Zeitabschnitt von 1892 bis 1931 dominierte die Zonalzirkulation mit einem Maximum um 1924/25, während die Meridionalzirkulation von 1892 bis 


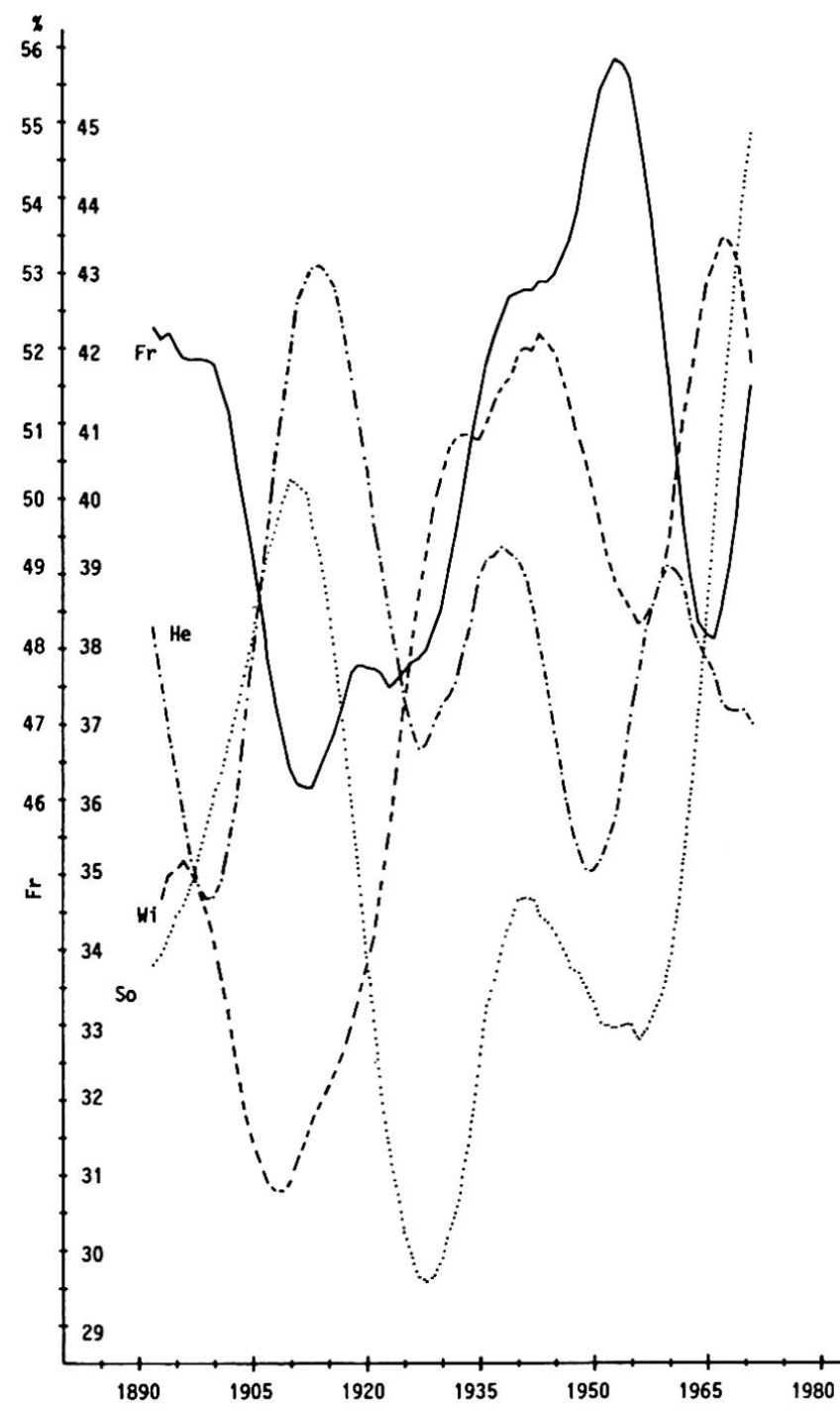

Abb. 2 Meridionalzirkulation (Jahreszeiten)

1934 unterdurchschnittlich häufig auftrat und 1925 ihr absolutes Minimum einnahm. Die Vorherrschaft der zonalen Jahreszirkulation erklärt sich in erster Linie durch die übernormalen Häufigkeitswerte im Frühjahr (1895 bis 1935) und im Winter (1896 bis 1925) bei einer gleichzeitig unter dem Durchschnitt liegenden meridionalen Frühjahrs- (1902 bis 1934) und Winterzirkulation (1892 bis 1924). AnschlieBend kehrten sich die Zirkulationsverhältnisse um. Von 1935 bis 1971 lag die relative Jahreshäufigkeit der Meridionalzirkulation (Maximum: 1971) über, die der Zonalzirkulation (Minimum: 1960) von 1932 bis 1971 unter dem langjährigen Mittel. Die jahreszeitlichen Häufigkeitsentwicklungen in dieser Epoche ließen insbesondere eine Dominanz meridionaler Wetterlagen im Frühjahr (1935 bis 1960) und im Winter (1925 bis 1971) erkennen sowie minimale Häufigkeitswerte zonaler Wetterlagen im Frühjahr (1936 bis 1971) und im Winter (1926 bis 1971). Die Steigerung der meridionalen Zirkulation in den

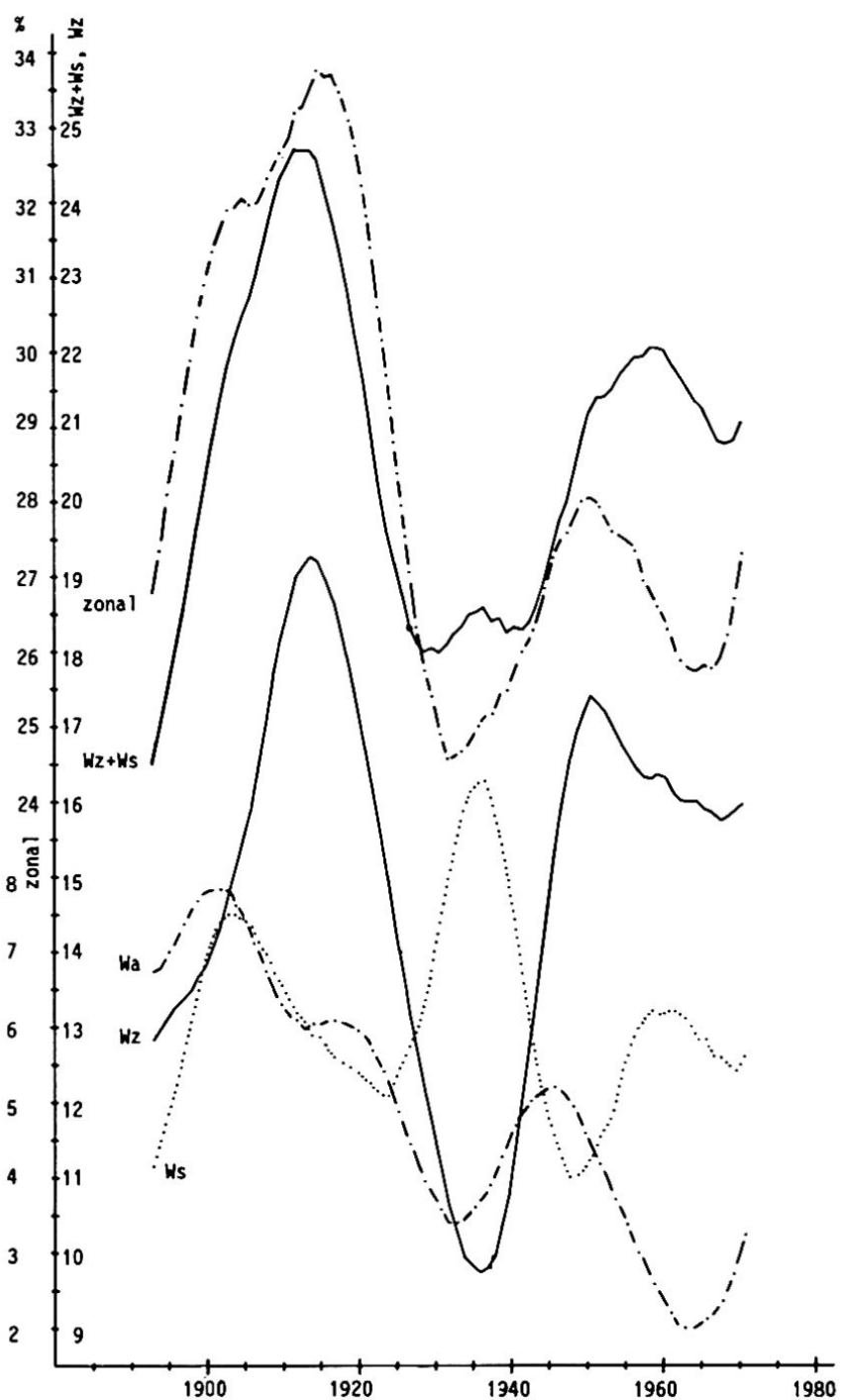

Abb. 3 Zonalzirkulation (Winter)

letzten Jahren erfolgte besonders auf Kosten der gemischten Zirkulation, die seit 1958 eine markante Abnahme von $33,3 \%$ auf $28,5 \%$ im Jahre 1971 zeigte. Diese Erscheinung ist im Herbst und im Winter kaum ausgebildet, im Frühjahr tritt sie seit 1964/ 66 auf, und ganz besonders im Sommer ist seit 1928/ 34 eine der jährlichen Entwicklung analoge Änderung zu beobachten.

\section{Wetterlagenanalyse}

Das unterschiedliche klimatische Verhalten der Großwetterlagen zeigt sich deutlich in den Wirkungen auf die Temperatur und auf andere Klimaelemente. Es ist eine Folge der durch die Luftströmungen zugeführten Luftmassen mit unterschiedlicher Herkunft und dementsprechend mit unterschiedlichen Eigenschaften. Jede Großwetterlage führt be- 
stimmte Luftmassen heran, die ihrerseits ihre Auswirkungen im Wettergeschehen haben. Die mit den Luftmassen verknüpften Wettererscheinungen erlauben es, daß durch die Häufigkeit von Luftmassen bzw. Großwetterlagen der Gang derWitterung, also das Klima, charakterisiert werden kann.

Großwetterlagen zeigen in der Häufigkeit einen mehr oder weniger deutlich ausgeprägten Jahresgang. Neben Häufigkeitsänderungen im Jahresgang bestehen über größere Zeiträume hinweg säkulare Schwankungen der Häufigkeiten bestimmter Zirkulationsformen bzw. der zugehörigen Großwetterlagen (vgl. Punkt 2).

Im Hinblick auf den säkularen Temperaturgang ergibt sich hieraus die Fragestellung nach Zusammenhängen desselben mit den säkularen Schwankungen der Zirkulation. Zur Klärung des Sachverhaltes wurden Häufigkeiten der Großwetterlagen und Großwettertypen in Beziehung zu den jährlichen und jahreszeitlichen Temperaturen gesetzt. Ziel dieser Wetterlagenanalyse ist, die regionalen Auswirkungen der verschiedenen Wetterlagen auf die Temperaturverteilung herauszuarbeiten und so zur Erklärung raumzeitlicher Unterschiede des Klimas beizutragen. Die raumzeitliche Analyse der wichtigsten Wetterlagen klärt, wie sich ihre Wetterwirksamkeit überhaupt äußert und ob und in welcher Art es wetterlagenbedingte regionale Unterschiede in der Temperaturverteilung gibt.

Die Untersuchung ergab, daß insbesondere im Herbst oder im Winter das überdurchschnittlich häufige Auftreten des Süd-, Südwest-, West-GTund der Wa, Wz und Wz+Ws in Mitteleuropa zu signifikanten Temperaturüberschüssen führt, das des Nord-, Ost-GT und der meridionalen Großwetterlagen hingegen mit negativen Temperaturanomalien verknüpft ist (vgl. Abb. 4/Tab. 1).

\section{Säkulare Temperaturschwankungen und Häufig- keiten der Großwetterlagen und Großwettertypen}

Mit Hilfe der Wetterlagenanalyse wird versucht, einen Zusammenhang aufzuzeigen zwischen Klimaschwankungen und Häufigkeitsänderungen typischer Wetterlagen. Es wird untersucht, inwieweit die Haupttendenzen im säkularen Temperaturverlauf mit den Häufigkeitsschwankungen bestimmter Großwetterlagen und Großwettertypen in Verbindung gebracht, und die Klimaschwankungen durch geänderte Zirkulationsverhältnisse erklärt werden können.

An dieser Stelle wird über die Zirkulations- und Temperaturschwankungen in bestimmten Jahreszeiten berichtet.

Frühjahr: Um 1850/53 erreichen die Frühjahrsmitteltemperaturen in Mitteleuropa ihr absolutes Mini- mum. Im Süden und Südosten des Untersuchungsgebietes liegen zu diesem Zeitpunkt die Temperaturen $0,9^{\circ} \mathrm{C}$ bis $1,2^{\circ} \mathrm{C}$ unter den langjährigen Mittelwerten, während im deutsch-holländischen Bereich die Frühjahre nur um $0,6^{\circ} \mathrm{C}$ bis $1,0^{\circ} \mathrm{C}$ zu kühl sind. Nach dem Tiefstwert von 1850/53 zeigen die Temperaturkurven einen Anstieg von $1,1^{\circ} \mathrm{C}$ bis $1,2^{\circ} \mathrm{C}$ bis zum Höchstwert 1946/49 und überschreiten damit die 100jährigen Mittel $1881 / 1980$ um $0,5^{\circ} \mathrm{C}$ bis $1,1^{\circ} \mathrm{C}$. Die Normalwerte werden erst $1904 / 19$ übertroffen.

Diese Entwicklung geht zunächst mit Zunahmen der zonalen und gemischten Frühjahrszirkulation einher, die sich im Zeitraum 1892 bis zum Maximum $1923 / 24$ (1911) von $20,9 \%$ auf $24,0 \%$ bzw. von $25,5 \%$ auf $30,1 \%$ erhöhen. Die Meridionalzirkulation hingegen verringert sich im Zeitabschnitt 1892 bis 1913 von $52,3 \%$ auf $46,2 \%$. Die mitteleuropäischen Zirkulationsverhältnisse sind im weiteren Verlauf durch abwechselnd gegenläufige Tendenzen gekennzeichnet: Der Abnahme der zonalen und gemischten Zirkulation bis 1954 (1940/41) auf 16,8\% $(25,7 \%)$ steht eine Erhöhung der meridionalen bis 1953 auf $55,9 \%$ gegenüber. Obwohl zwischen den meisten untersuchten Großwetterlagen und den Frühjahrstemperaturen keine Beziehung festgestellt werden konnte, läßt sich der Anstieg der Frühjahrsmittelwerte vermutlich mit einer Häufigkeitsabnahme der Kälterückfälle und mit einer $\mathrm{Zu}$ nahme von Wetterlagen warmen Charakters in Verbindung bringen. Die Ost- und Nordostlagen weisen seit 1892 eine rückläufige Tendenz auf, und insbesondere ab 1922 (1919) treten sie nur noch unterdurchschnittlich häufig auf. Auch die Nordwestund Nordlagen nehmen ab und bleiben seit 1919 (1892) unter der Norm. Dagegen nimmt die Häufigkeit der Südwest- und Südströmungen seit 1911 (1916) wieder zu. Trotz des oben skizzierten säkularen Ganges der Zonalzirkulation (Abnahme seit 1923/24!) kann in etwa im Zeitraum desTemperaturanstiegs bis 1946/49 ein überdurchschnittlich häufiges Vorkommen der einzelnen zonalen Großwetterlagen als auch der gesamten zonalen Frühjahrszirkulation festgestellt werden. So ist für die Wa von 1911 bis 1930 und von 1943 bis 1956, für die im Frühjahr zeitweise schwül-warm in Erscheinung tretende Ws von 1921 bis 1940 und für die zyklonalen Westlagen Wz+Ws von 1897 bis 1915 und von 1920 bis 1944 ein überdurchschnittlich häufiges Auftreten nachzuweisen. Der Temperaturüberschüsse bewirkende W-GT bleibt im Zeitabschnitt 1895 bis 1935 über der Norm. Auch die Großwetterlagenkombination HM+BM ist von 1906 bis 1956 überdurchschnittlich häufig vertreten. Hier ergab die Untersuchung, daß das gehäufte Vorkommen des GT $\mathrm{HM}+\mathrm{BM}$ in den Niederlanden, im nord- und mitteldeutschen Bereich und in der Schweiz zu signifikanten positiven Temperaturanomalien führt. Der Anstieg der Frühjahrstemperaturen dürfte somit wohl 


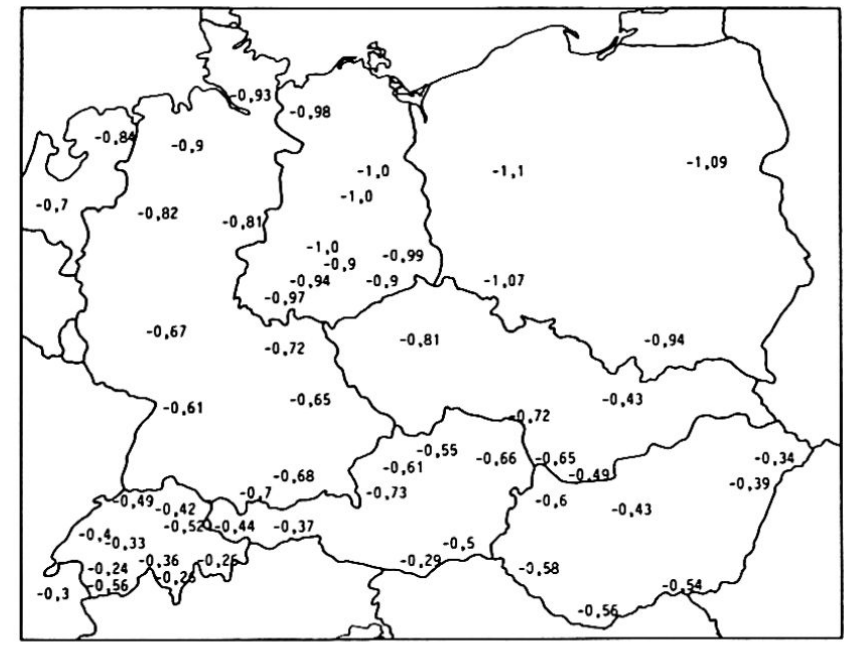

Abb. 4a Absolute Abweichung (in ${ }^{\circ} \mathrm{C}$ ) des wetterlagenabhängigen Mittelwertes (meridionale Zirkulationsform) vom langjährigen Mittelwert der Wintermitteltemperaturen

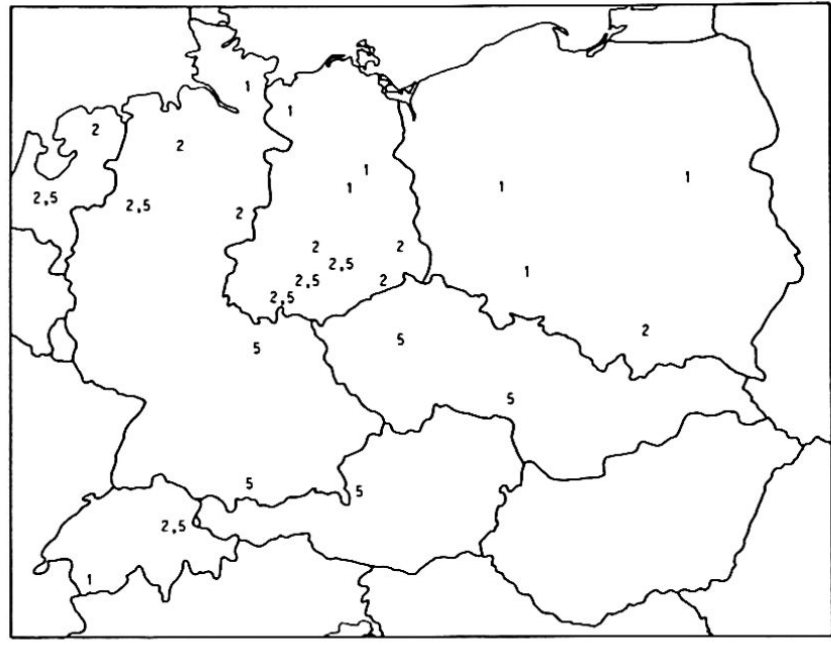

Abb. 4b Irrtumswahrscheinlichkeiten in Prozent für das Auftreten der Differenzen zwischen wetterlagenabhängigen (meridionale Zirkulationsform) und langjährigen Mittelwerten der Temperaturen in erster Linie auf die mit dem 20. Jahrhundert beginnende Belebung der zonalen Zirkulation und des Hochdruck-Großwettertyps zurückzuführen sein. Nach dem Temperaturmaximum 1946/49 setzt bis zum Beobachtungsende im Untersuchungsgebiet ein Temperaturrückgang von $0,2^{\circ} \mathrm{C}$ bis $1,4^{\circ} \mathrm{C}$ ein. In den meisten Fällen wird sogar der Normalstand wieder unterschritten. H. v. RUDLOFF (1967, S. 172) sieht die Ursache der rückläufigen Tendenz in der Verlagerung der Hochdruckzentren nach Westen, Nordwesten und Norden. Seit 1948 (1950) nehmen Ostund Nordwestlagen wieder zu, während Hochdrucklagen seit 1947 abnehmen. Bereits ab 1957 stellen sich für den GT HM + BM unterdurchschnittliche Häufigkeitswerte ein. Die Zonalzirkulation, die $1953 \mathrm{ihr}$ absolutes Minimum einnimmt, bleibt in dieser Epoche unter dem Durchschnitt.

Sommer: Der Verlauf der Sommertemperaturkurven ist seit Beobachtungsbeginn bis 1914/24 fallend. Besonders ausgeprägt ist dieser Temperaturrückgang seit dem absoluten Maximum 1792/93 bei den Säkularreihen im Südosten $\left(1,6-1,9^{\circ} \mathrm{C}\right)$. Einer letzten leichten Erwärmungsphase von 1885/91 bis 1898/1902 schließt sich die Abnahme der Mitteltemperaturen zum absoluten Minimum 1914/24 an. In der Schweiz und in Österreich wird der Tiefstwert 1914/17 erreicht, im deutschen Bereich 1917/21, im Südosten des Untersuchungsgebietes 1916/20 und in Polen 1922/24.

Die Periode abnehmender Sommermitteltemperaturen bis 1914/24 weist durchweg bis 1924 unterdurchschnittliche Häufigkeitswerte der Hochdrucklagen, bis 1956 der Ost- und bis 1932 der Nordostlagen auf bzw. eine Zunahme der Nordwest- und Nordlagen bis 1922 (1909). Im Zeitabschnitt 1892 bis 1940 (1892 bis 1922) treten die Großwettertypen Nordwest und Nord überdurchschnittlich häufig auf. Auch die zonale Sommerzirkulation liegt von 1892 bis 1909 über dem Durchschnitt. Obwohl zwischen den in besonders großer Häufigkeit auftretenden Nordwest-, Nord- und Westlagen und den durch sie hervorgerufenen Temperaturdefiziten keine gesicherte Beziehung festgestellt werden konnte, dürfte der Rückgang der Sommertemperaturen durch ein Minimum an Hochdruck-, Ost- und Nordostlagen und ein Maximum an Nord-, Nordwest- und Westlagen zu erklären sein. Auch D. KLAUS (1984, S. 161) führt aus, daß die Sommertemperaturen näherungsweise der Häufigkeitsentwicklung der ozeanisch geprägten Großwetterlagen folgen (Wz, Ws, Ww, NWa, NWz, Na, HNa, HB, Nz, HNz, TrM). Die Zonalzirkulation nimmt im Sommer mit 30,4\% ihr absolutes Maximum ein. Trotz großer Häufigkeit konnte keine Beeinflussung der Mitteltemperaturen festgestellt werden. Auch J. wUSTElT (1962, S. 52) findet für die Jenaer Temperaturen keinen $\mathrm{Zu}$ sammenhang zwischen überdurchschnittlicherWestwettertätigkeit und den Monatsmitteltemperaturen im Mai, Juni, Juli und August.

Mit der Temperaturverwerfung um 1914/24 und einer Zunahme von $0,9^{\circ} \mathrm{C}$ bis $1,5^{\circ} \mathrm{C}$ bis zum Höchstwert 1936/53 kehren sich die Zirkulationsverhältnisse um und verursachen den Anstieg der Sommermitteltemperaturen. Ab 1923/30 werden die Sommer in Mitteleuropa warm, und mit dem Sommeroptimum verzeichnet man einen Temperaturüberschuß von $0,5^{\circ} \mathrm{C}$ bis $1,1^{\circ} \mathrm{C}$. Die Hochdruckwetterlagen (seit 1909), Ost- (seit 1927), Nordost- (seit 1925) und Südlagen (seit 1934) zeigen eine Häufigkeitszunahme mit einem Maximum um 1940/47 (für die Ostlagen: 1970). Insbesondere im niederländi- 
schen und deutschen Raum und für den GT $\mathrm{HM}+\mathrm{BM}$ und den E-GT auch im schweizerischen und westlichen österreichischen bzw. im tschechischen Bereich kann ein Zusammenhang mit dem Vorherrschen dieser Großwetterlagen - außer für den S-GT - im Zeitraum 1925/33 bis 1952/71 und dem Temperaturanstieg als gesichert gelten. Gleichzeitig schwächt sich die zonale Zirkulation ab (seit 1927) und unterschreitet 1934 bis 1942 die mittlere relative Häufigkeit von $30,4 \%$. Auch die Nord- und Nordwestlagen sind bis 1961/63 (1950) rückläufig. Nach 1936/53 zeichnet sich das Ende des Klimaoptimums ab. Die Sommertemperaturen sind rückläufig. Allerdings bleiben im Einflußbereich des GT $\mathrm{HM}+\mathrm{BM}$, der Ost- und Nordostlagen, die ja bis 1952/71 vorherrschend sind, in dieser Jahreszeit die Temperaturen bis zum Beobachtungsende über dem Durchschnitt. In den übrigen mitteleuropäischen Bereichen werden die Sommer ab 1951/64 zu kühl. Der Temperaturrückgang ist von einem Anstieg der nördlichen (seit 1961/63) und nordwestlichen (seit 1949) Wetterlagen begleitet, während Hochdrucklagen seit 1947 abnehmen und seit 1961 nur noch unterdurchschnittlich häufig auftreten.

In der vorausgegangenen Untersuchung konnte zwischen den jahreszeitlichen Temperaturschwankungen mitteleuropäischer Stationen und der Häufigkeitsentwicklung bestimmter Großwetterlagen enge Beziehungen festgestellt und in ihrem zeitlichen Verlauf verfolgt werden. Im Frühjahr bestehen Zusammenhänge der Mitteltemperaturen mit dem GT HM + BM, im Sommer darüber hinaus auch mit dem E- und NE-GT.

\section{Summary}

The present paper is an attempt to contribute to the research of historical climatology of Central Europe. The representation and working out of the spatial and temporal differentiation of the secular variations in temperature occurring in Central Europe in the last one hundred or two hundred years were to the fore of the study. By means of the Gaussian low-pass filtering method the mean air temperatures for months, years and seasons have been calculated and graphically represented for 42 stations. The analysis of the climatic element showed extensive variations. The variations in climate which have occurred are primarily caused, as has been proved, by changes in the general weather situation of Central Europe. Frequency counts of the general weather situations for seasons and years in the period from 1881 to 1982 conveyed a very differentiated picture of the variations of the circulation structure above Europe during this observation period. Furthermore the study was to clarify the question to what degree the observed circulation variations would explain the secular march of temperature.
First it was necessary to relate frequencies of the general weather situations and general weather types to the annual and seasonal temperatures and thus to make clear the regional effects of the different weather situations on the temperature circumstances. Thus the main tendencies in the secular march of temperature could be connected with the frequency variations of certain general weather situations and general weather types respectively, and the variations in climate could be explained by changed circulation conditions.

\section{Literatur}

BREZOWSKY, H. (1952): Säkulare Schwankungen der Zirkulation. - Berichte des Deutschen Wetterdienstes in der USZone, Nr. 35, 1952, S. 48-56, Bad Kissingen.

EISENHARDT, T. (1968): Klimaschwankungen im RheinMain-Gebiet seit 1880. - Forschungen zur Deutschen Landeskunde, Bd. 165, 1968, Bad Godesberg.

GRABAU, J. (1985): Klimaschwankungen und Großwetterlagen in Mitteleuropa seit 1881 (2 Bde.), Paderborn 1985.

HESS, P. \& BREZOWSKY, H. (1977): Katalog der Großwetterlagen Europas (1881-1976). - Berichte des Deutschen Wetterdienstes, 3. verbesserte und ergänzte Auflage, Nr. 115, Bd. 15, 1977, Offenbach am Main.

HUPFER, P. (1962): Beitrag zur Kenntnis langjähriger Zirkulationsschwankungen über Mitteleuropa und ihres Zusammenhanges mit den säkularen Änderungen der Lufttemperatur. - Wissenschaftliche Zeitschrift der Karl-Marx-Universität Leipzig, Mathematisch-Naturwissenschaftliche Reihe, Jg. 11, 1962, H. 2, S. 245-251, Leipzig.

KLAUS, D. (1984): Hat sich das Klima Mitteleuropas geändert? 100 Jahre europäische Großwetterlagenstatistik. Ber. zur deutschen Landeskunde, Bd. 58, Heft 1, 147-182, Trier.

LALLA, H. (1976): Klimaschwankungen im Bereich der Iberischen Halbinsel seit 1880, Bochum 1976.

MEYER, A.-M. (1978): Klimaschwankungen im maritimen und kontinentalen Raum Europas seit 1871. - Bochumer Geographische Arbeiten, H. 32, Paderborn 1978.

RUDLOFF, H. v. (1967): Die Schwankungen und Pendelungen des Klimas in Europa seit dem Beginn der regelmäßigen Instrumenten-Beobachtungen (1670). - Sammlung "Die Wissenschaft”, Bd. 122, Braunschweig 1967.

SCHERHAG, R. (1936): Die Zunahme der atmosphärischen Zirkulation in den letzten 25 Jahren. - Annalen der Hydrographie und maritimen Meteorologie, Jg. 64, 1936, H.9, S. 397-407, Berlin.

WAGNER, A. (1929): Untersuchungen der Schwankungen der Allgemeinen Zirkulation. - Geografiska Annaler, Bd. 11, 1929, S. 32-88, Stockholm.

WMO (World Meteorological Organization) (1966): Climatic Change. Technical Note No. 79, Geneva.

WUSTELT, J. (1962): Die Temperaturschwankungen in der Klimareihe von Jena und ihre Beziehungen zur Witterungskunde und zur Klimageschichte Europas. - Abh. d. Meteorol. u. Hydrolog. Dienstes der DDR, Nr. 66, Bd. IX, Berlin. 